\title{
SSupporting Information
}

\section{Electrochemical Water Oxidation of Ultra-Thin Cobalt Oxide-Based Catalyst Supported onto Aligned ZnO Nanorods}

Nandanapalli Koteeswara Reddy ${ }^{1, *, \#}$, Stefanie Winkler ${ }^{2}$, Norbert Koch $^{2}$, Nicola Pinna ${ }^{1, *}$

1 - Humboldt-Universität zu Berlin, Institut für Chemie, Brook-Taylor-Str. 2, 12489 Berlin, Germany

2 - Institut für Physik \& IRIS Adlershof, Humboldt-Universität zu Berlin, Brook-TaylorStrasse 6, 12489 Berlin, Germany

Corresponding Author Email: nandanak@cms.hu-berlin.de, nicola.pinna@hu-berlin.de 


\section{ADDITIONAL INFORMATION:}
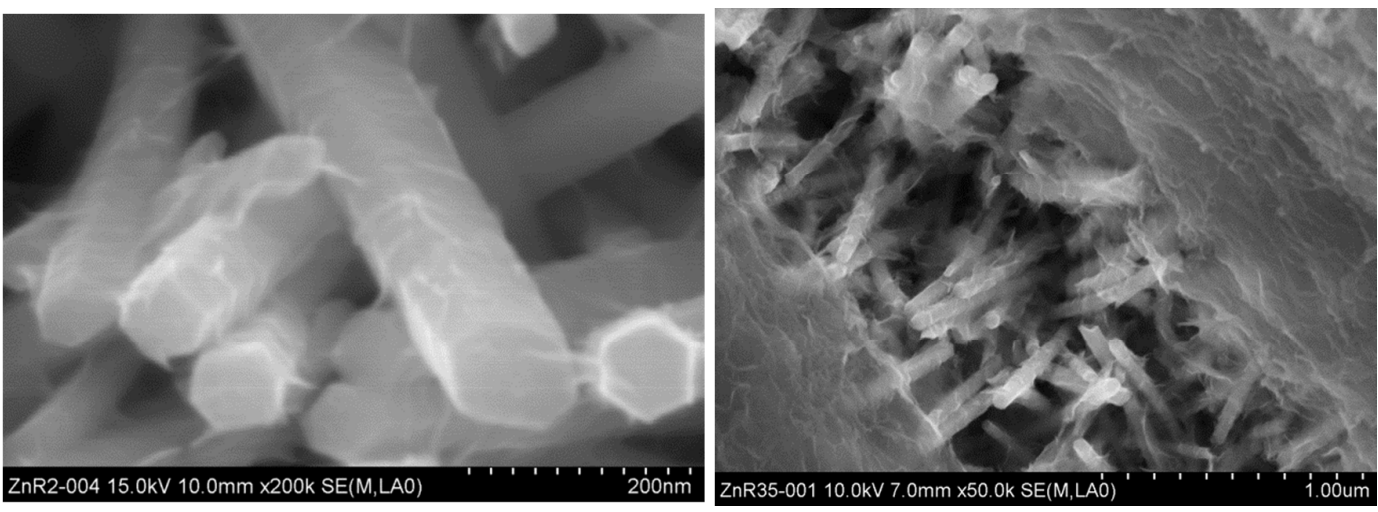

Figure S1: High magnification FESEM image of 30 and $180 \mathrm{~s} \mathrm{CoO}$ deposited ZnO NRs.

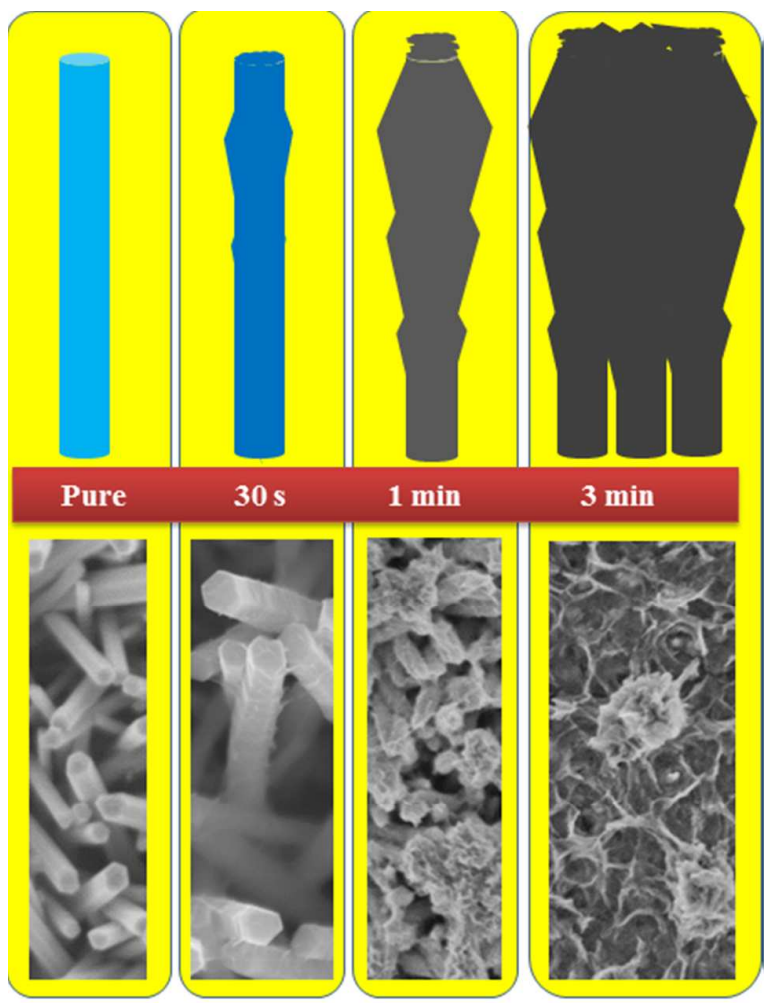

Figure S2: Change of growth morphology of $\mathrm{CoO}$ layer on $\mathrm{ZnO}$ NRs/FTO structures

(Possible growth mechanism of 30, 60, and $180 \mathrm{~s} \mathrm{CoO}$ deposited ZnO NRs/FTO structures). 

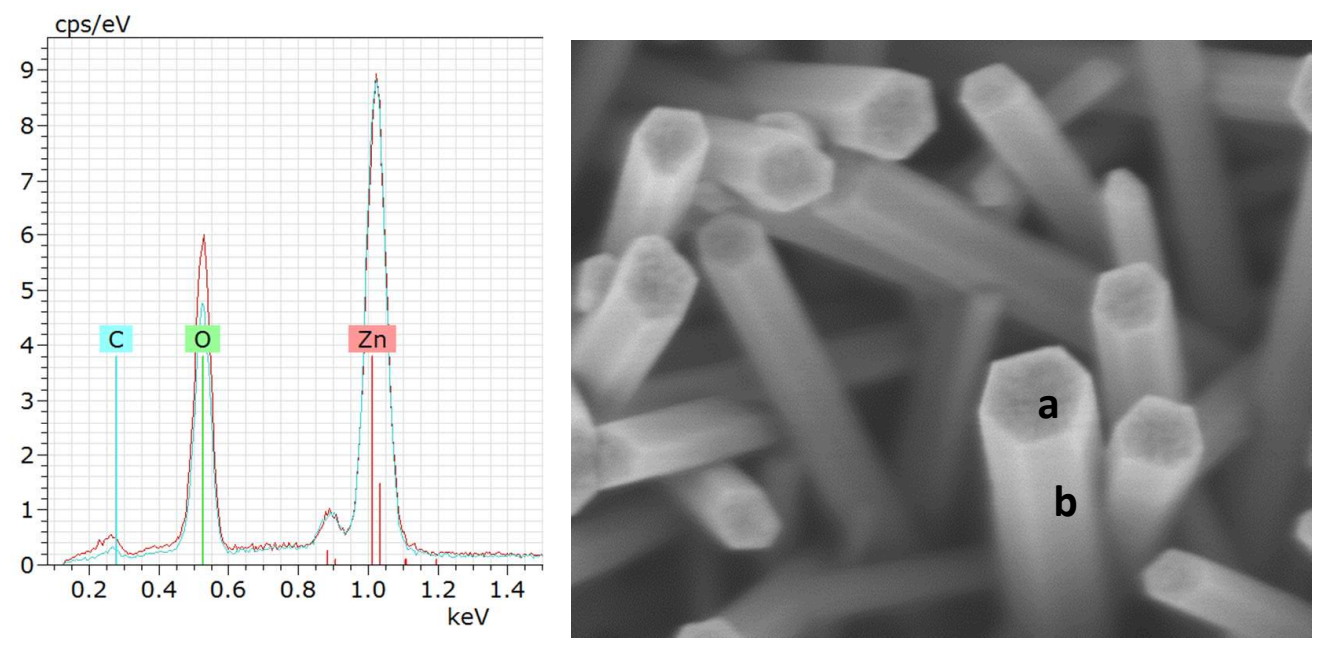

Figure S3: EDS spectra recorded at different places on pure $\mathrm{ZnO}$ NRs.

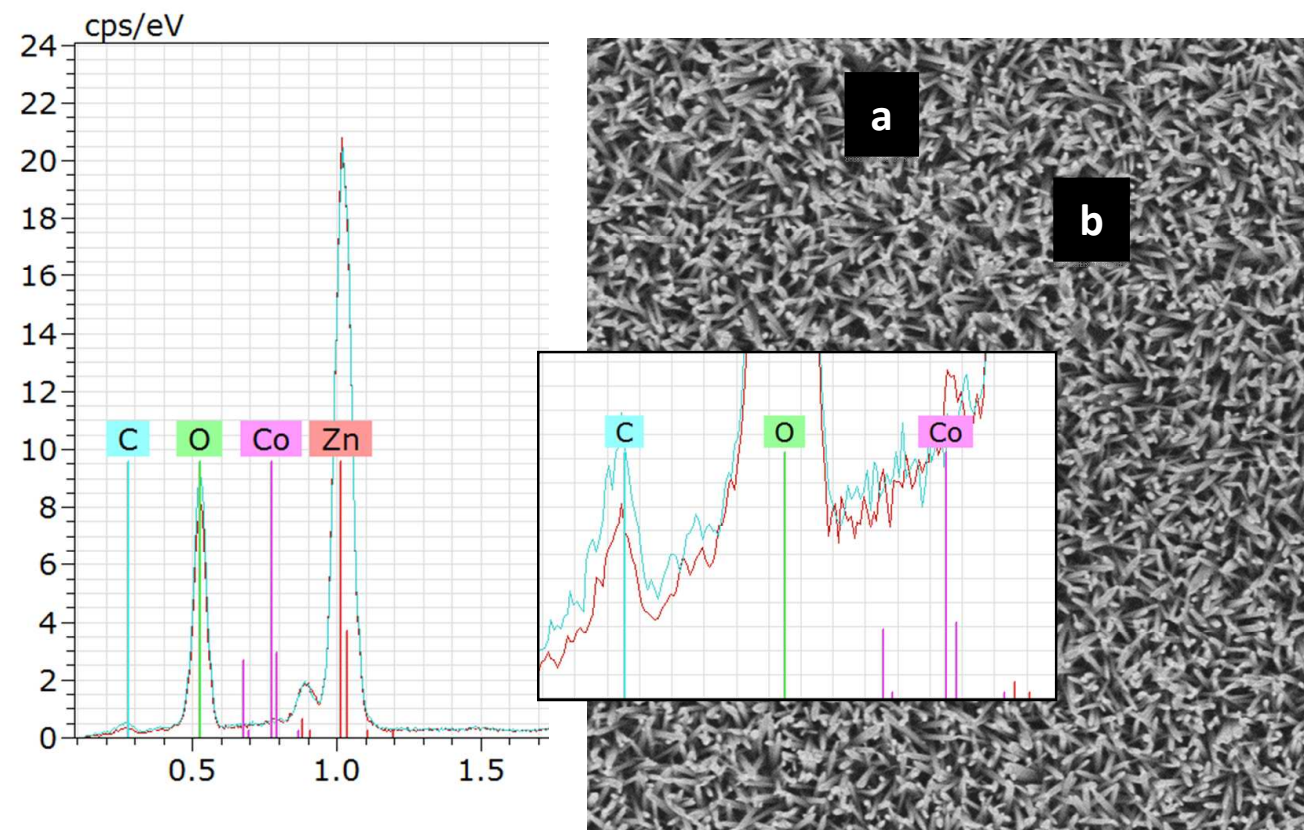

Figure S4: EDS spectra recorded at different places on 30 s CoO deposited ZnO NRs 

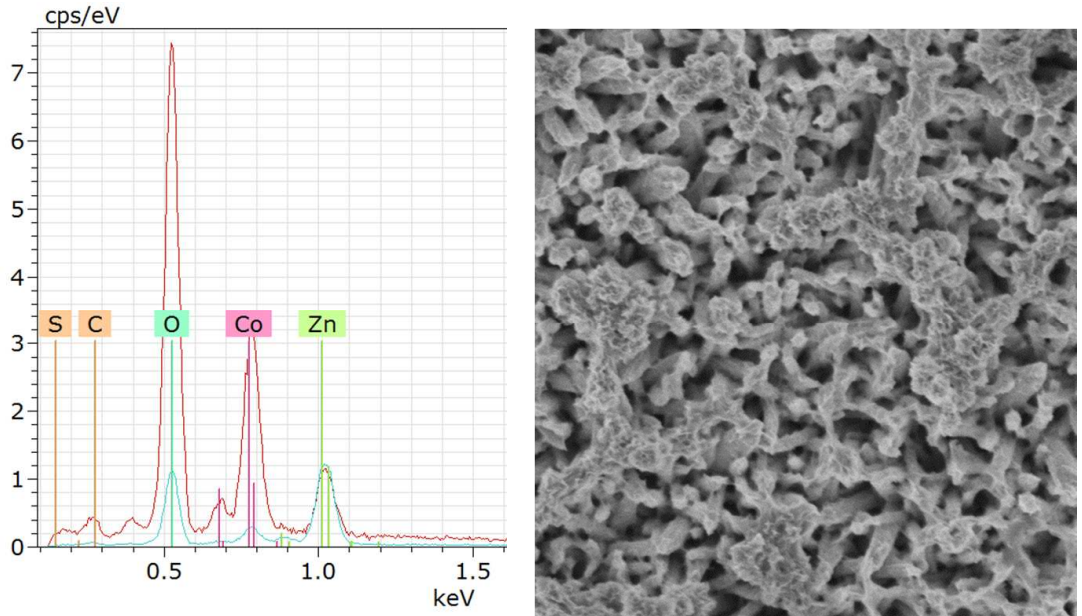

Figure S5: EDS spectra recorded at different places on $60 \mathrm{~s} \mathrm{CoO}$ deposited $\mathrm{ZnO}$ NRs
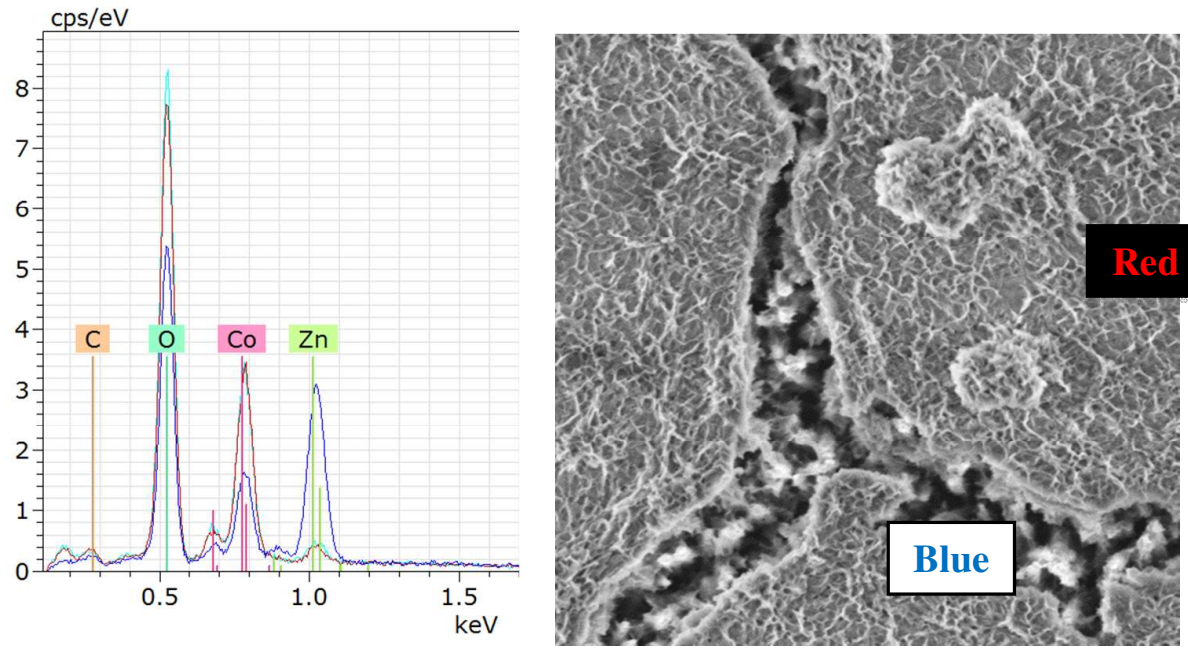

Green

Figure S6: EDS spectra recorded at different places on $180 \mathrm{~s}$ CoO deposited $\mathrm{ZnO}$ NRs

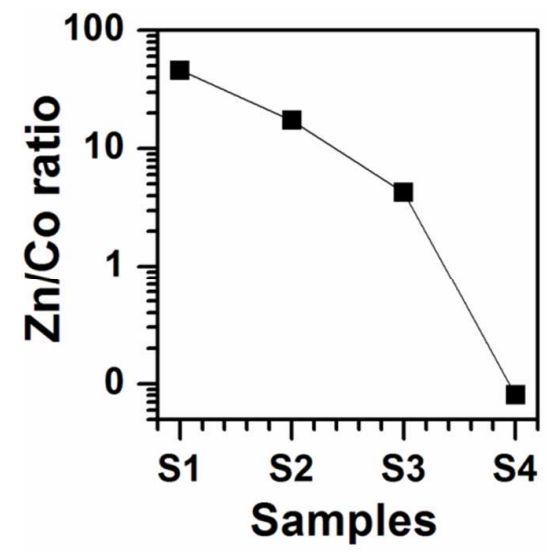

Figure S7: Variation of the surface $\mathrm{Zn} / \mathrm{Co}$ ratio of pristine and $\mathrm{CoO}$ deposited $\mathrm{ZnO}$ NRs 


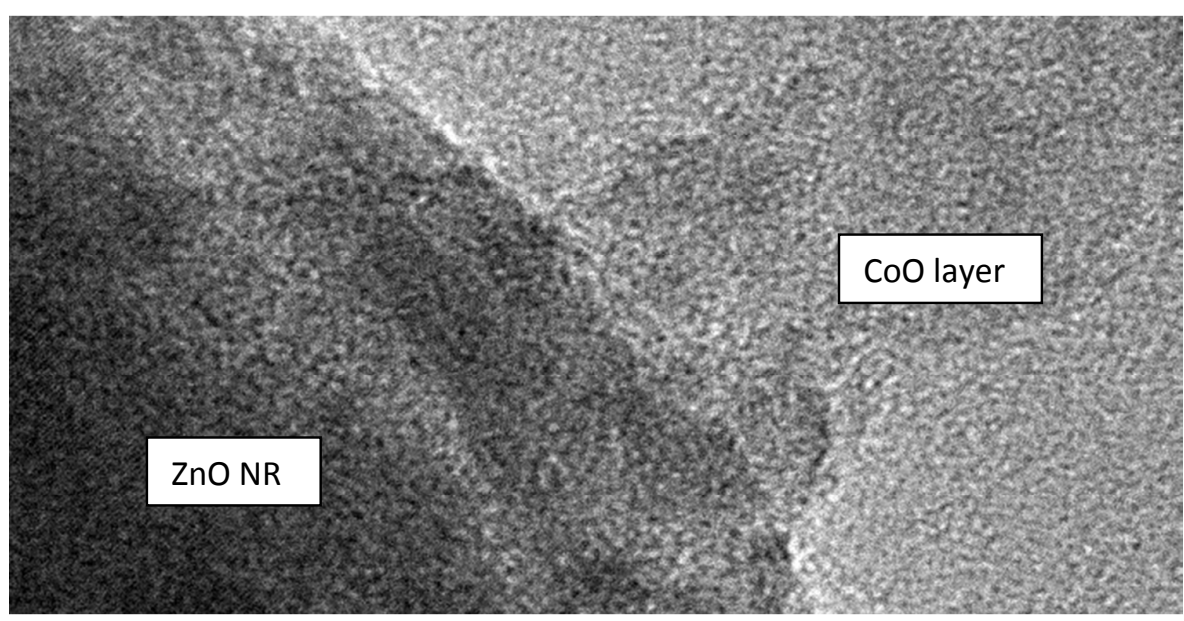

Figure S8: HRTEM image of $30 \mathrm{~s} \mathrm{CoO}$ deposited ZnO NR

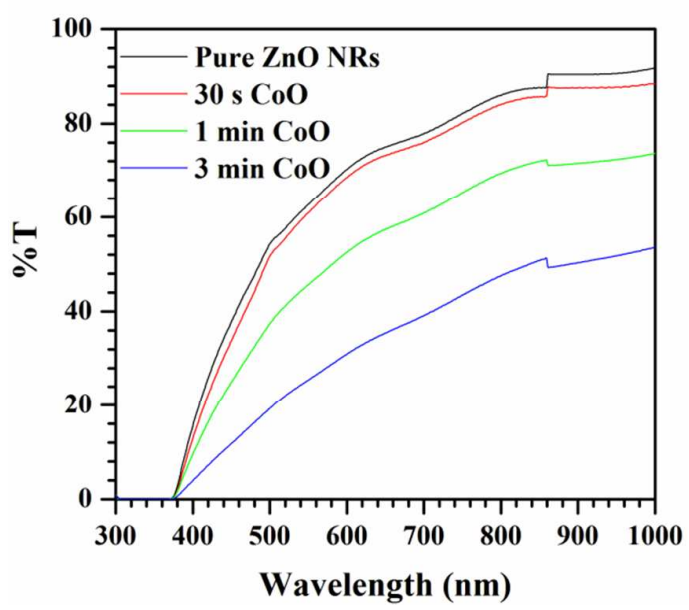

Figure S9: UV-Vis-NIR transmittance (\% $\%$ ) spectra of pure and CoO-deposited ZnO NRs.

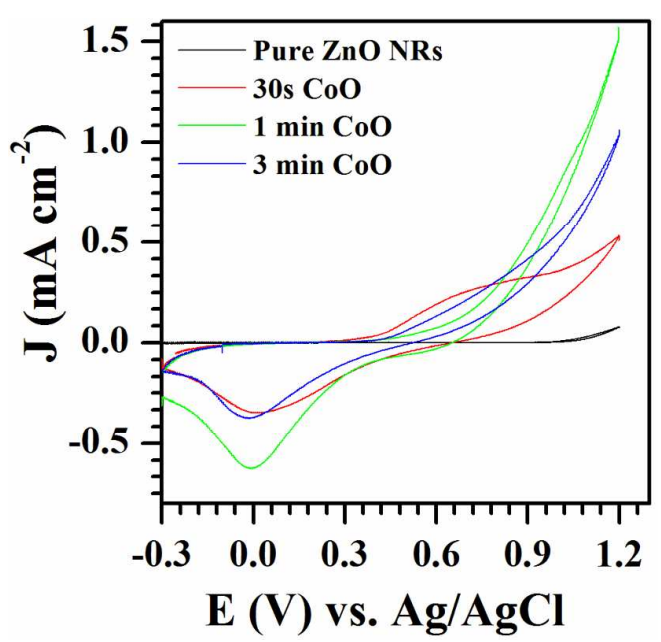

Figure S10: CVs of pure and $\mathrm{CoO}$ deposited $\mathrm{ZnO} \mathrm{NRs}$ 

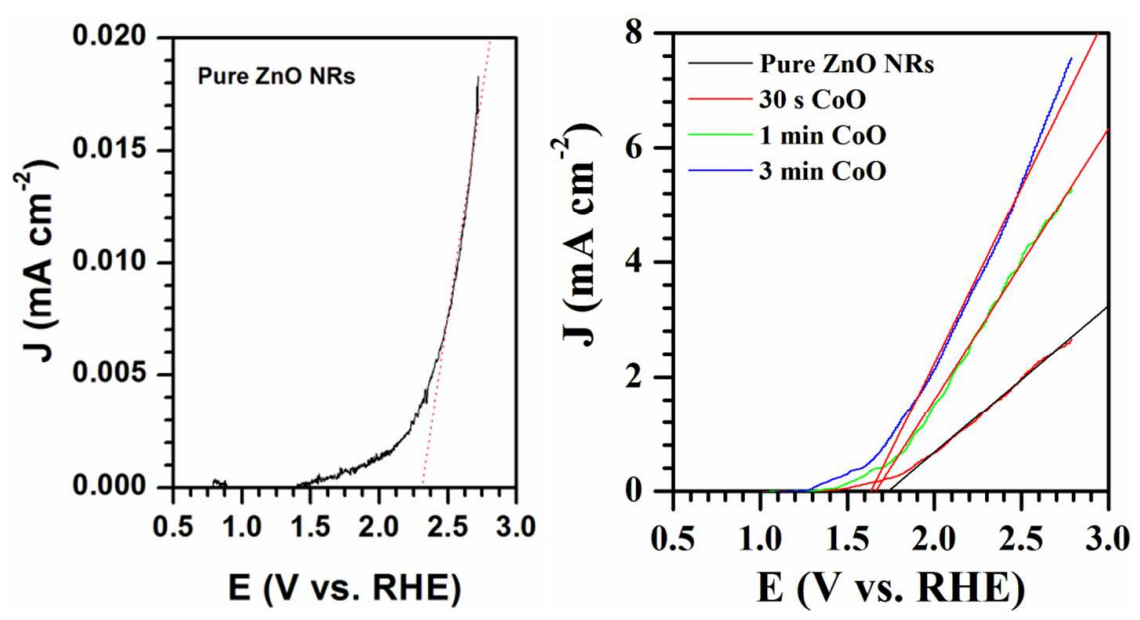

Figure S11: J vs E plots of pure and $\mathrm{CoO}$ deposited $\mathrm{ZnO}$ NRs: Evaluation of onset potentials and series resistance of OER devices (Onset potential of pure ZnO NRs is $2.31 \mathrm{~V}$ vs. RHE)

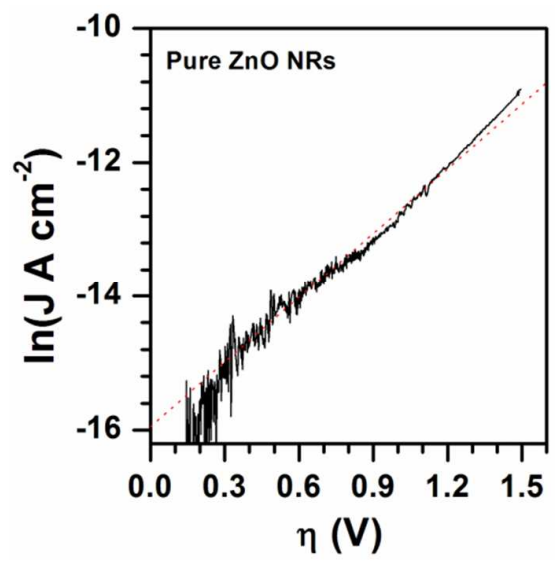

Figure S12: Tafel plot of pure ZnO NRs (Slope and intersection of the $\ln (\mathrm{J})$ vs $\eta(\mathrm{V})$ plot is 3.29 and -16.03 , respectively). 